\title{
SECOND ORDER NONLINEAR RANDOM DIFFERENTIAL EQUATIONS
}

\author{
B. C. Dhage, S. V. Badgire And J. Henderson
}

\begin{abstract}
In this paper, an existence result for a nonlinear second order ordinary random differential equation is proved under a Carathéodory condition. Two existence results for extremal random solutions are also proved for Carathéodory as well as discontinuous cases of the nonlinearity involved in the equations. Our investigations are placed in the Banach space of continuous real-valued functions on closed and bounded intervals of the real line together with an application of the random version of the Leray-Schauder principle.
\end{abstract}

Mathematics subject classification (2010): 35F05, 47H10, 47H40, 47N20, 60H25.

Keywords and phrases: initial value problem, random differential equation, random fixed point theorem, existence theorem, extremal solutions.

\section{REFERENCES}

[1] A. T. Bharucha-Reid, On the theory of random equations, Proc. Sympos. Appl. Math., Amer. Math. Soc., Providence, R.I., Vol. XVI (1964), 40-69.

[2] A. T. Bharucha-Reid, Random Integral Equations, Academic Press, New York, 1972.

[3] K. Deimling, Nonlinear Functional Analysis, Springer Verlag, New York, 1985.

[4] B. C. DHAGE, Monotone iterative technique for Carathéodory theory of nonlinear functional random integral equations, Tamkang J. Math., 33, 4 (2002), 341-351.

[5] B. C. DHAGE, Some algebraic and topological random fixed point theorems with applications to nonlinear random integral equations, Tamkang J. Math., 35 (2004), 321-345.

[6] B. C. DHAGE, A random version of a Schaefer type fixed point theorem with applications to functional random integral equations, Nonlinear Funct. Anal. Appl., 9 (2004), 389-403.

[7] P. Hans, Random fixed point theorems, Transactions of the first Prague Conference on Information Theory, Statistical Decision Functions, Random Process, (1957), 105-125.

[8] S. Heik Kilä AND V. Lakshmikantham, Monotone Iterative Techniques for Discontinuous Nonlinear Differential Equations, Pure and Applied Mathematics, Marcel Dekker, New York, 1994.

[9] C. J. Himmelbeg, Measurable relations, Fund. Math., 87 (1975), 53-72.

[10] S. Iтон, Random fixed point theorems with applications to random differential equations in Banach spaces, J. Math. Anal. Appl., 67 (1979), 261-273.

[11] G. S. Ladde and V. Lakshmikantham, Random Differential Inequalities, Academic Press, New York, 1980.

[12] V. LAKSHMikAnTHAM AND S. LeELA, Remarks on first and second order periodic boundary value problem, Nonlinear Anal., 8 (1984), 281-287.

[13] S. LEELA, Monotone method for second periodic boundary value problems, Nonlinear Anal., 7 (1983), 349-355.

[14] E. ZeIdLer, Nonlinear Functional Analysis and Its Applications: Part I, Springer Verlag, New York, 1985. 